\title{
EL ESQUEMA TRASCENDENTAL DE LAS \\ CATEGORÍAS DE LA CANTIDAD COMO \\ DETERMINACIÓN TEMPORAL
}

\section{THE TRANSCENDENTAL SCHEMA OF THE CATEGORIES OF QUANTITY AS TEMPORAL DETERMINATION}

\author{
MARTÍN ARIAS-ALBISU* \\ Consejo Nacional de Investigaciones Científicas y Técnicas, \\ Argentina
}

RESUMEN: El objetivo del presente artículo es mostrar que dos tesis acerca de la naturaleza de los esquemas trascendentales son válidas para el caso del esquema trascendental de las categorías de la cantidad. Semejantes tesis son: (1) los esquemas trascendentales son procedimientos de síntesis y al mismo tiempo las propiedades temporales fundamentales de los objetos empíricos producidas por tales procedimientos. En el caso del esquema trascendental que nos ocupa, por un lado, el esquema en tanto procedimiento es denominado por Kant «número» (Zahl). Por otro lado, sostenemos que el esquema en tanto producto viene dado por lo que Kant llama «magnitud extensiva» (extensive Größe). (2) Los esquemas trascendentales no son determinaciones del tiempo puro, sino determinaciones temporales de lo empírico. En otras palabras, las síntesis de los esquemas en tanto procedimientos no determinan la intuición pura, sino que determinan temporalmente la multiplicidad empírica.

PALABRAS ClaVE: Kant, Crítica de la razón pura, esquemas trascendentales, cantidad, procedimiento, producto, número, magnitud extensiva.

ABSTRACT: The objective of this article is to show that two theses about the nature of transcendental schemas are valid for the case of the transcendental schema of the

\footnotetext{
* Dirección postal: San Juan 1609 3 B, (2000) Rosario, Santa Fe, Argentina. E-mail: arias.martin@gmail.com
} 
categories of quantity. These theses are: (1) transcendental schemas are procedures of synthesis and, simultaneously, the main temporal properties of the empirical objects produced by these procedures. In the case of the transcendental schema that we are studying, on one hand, Kant calls the schema, insofar as it is a procedure, a "number," (Zahl). On the other hand, we hold that the schema as a product is determined by what Kant calls "extensive magnitude" (extensive Größe). (2) Transcendental schemas are not determinations of pure time, but temporal determinations of empirical things. In other words, the syntheses of schemas as procedures do not determine pure intuition but, rather, they temporally determine empirical multiplicity.

KeYWORDs: Kant, Critique of Pure Reason, transcendental schemata, quantity, procedure, product, number, extensive magnitude.

En este artículo efectuamos un análisis detallado de la concepción kantiana del esquema trascendental correspondiente a las categorías de la cantidad. Nos basaremos, principalmente, en la caracterización de ese esquema presentada en la Crítica de la razón pura. El objetivo de ese análisis es mostrar que dos tesis fundamentales sobre la naturaleza de los esquemas trascendentales son válidas para el caso del esquema trascendental que nos ocupa. Tales tesis son las siguientes: (1) Los esquemas trascendentales son, por un lado, procedimientos de determinación o síntesis que se originan en la imaginación y se dirigen a la multiplicidad empírica y, por el otro, las determinaciones o propiedades temporales fundamentales de los objetos empíricos producidas por semejantes procedimientos. Llamaremos a estos aspectos de los esquemas trascendentales, respectivamente, esquemas-procedimientos y esquemas-productos. Hemos defendido en otro trabajo esta novedosa tesis nuestra, aunque de una manera principalmente general, es decir, para el concepto de esquema trascendental en general. ${ }^{1}$ (2) Los esquemas trascendentales no son determinaciones del tiempo puro, sino determinaciones temporales de lo empírico. Esta tesis tiene una larga historia en la bibliografía crítica. Sin embargo, no conocemos ningún estudio que intente demostrarla con respecto al esquema trascendental que nos ocupa ${ }^{2}$. Cabe destacar que no analizaremos las dos tesis men-

\footnotetext{
${ }^{1}$ Cf. Arias Albisu (2010) y sección II.

${ }^{2}$ Cf. sección III.
} 
cionadas ni en su generalidad ni en todas sus implicancias. Por el contario, nos limitaremos a mostrar que son válidas para el caso particular examinado en este trabajo, a saber, el esquema trascendental de la cantidad.

Antes de comenzar nuestros análisis del esquema de las categorías de la cantidad, intentaremos que los mismos sean comprensibles para el lector por medio de una presentación general de la doctrina kantiana del esquematismo trascendental. El objetivo más general del capítulo de $K r V$ «Del esquematismo de los conceptos puros del entendimiento ${ }^{3}$ es determinar bajo qué condiciones debe tener lugar la aplicación de las diferentes categorías a la multiplicidad de las intuiciones empíricas dadas a la sensibilidad. Tal aplicación parece a primera vista impracticable en virtud de la heterogeneidad que se presenta entre las categorías y la multiplicidad empírica. Esta relación de heterogeneidad consiste en que a lo pensado en las categorías no le corresponde ningún elemento intuitivo. En efecto, el contenido de las categorías proviene enteramente del entendimiento y es completamente independiente de la intuición espacio-temporal humana. Por este motivo, las categorías no son conceptos limitados a los objetos empíricos dados a nuestra intuición, sino que permiten pensar objetos de una intuición en general no especificada.

La función de los esquemas trascendentales es establecer un vínculo de homogeneidad entre las distintas categorías y la multiplicidad empírica. Tal vínculo hace posible la subsunción de la multiplicidad empírica bajo esas categorías o, en otras palabras, la aplicación de las segundas a la primera ${ }^{4}$. Mediante esta aplicación, la multiplicidad empírica se constituye como objeto empírico de una experiencia humana única.

${ }^{3}$ A 137/B 176 ss. Remitimos a la Crítica de la razón pura (Kritik der reinen Vernunft $=$ KrV) según la paginación de la edición original («A» hace referencia a la primera edición de 1781, y «B» a la segunda de 1787). Citamos esta obra de acuerdo con la traducción de M. Caimi (Kant, 2007). En cuanto a las fuentes, nos hemos servido de los textos incluidos en Kant (1902 ss.) Remitimos a esta edición de conjunto con la sigla "AA» (Akademie-Ausgabe) y damos a continuación el número de tomo (en romanos) y de página (en arábigos). Cuando hacemos referencia a la sección de una obra sin mencionar esta última, ella pertenece a la Crítica de la razón pura (por ejemplo: «Axiomas de la intuición»).

${ }^{4}$ Hemos estudiado la concepción kantiana de la homogeneidad en Arias Albisu (2009). 
En la sección siguiente iniciaremos el análisis del esquema trascendental de las categorías de la cantidad. Sostendremos que la imaginación produce un único esquema para estas tres categorías (unidad, pluralidad y totalidad). Concluiremos que el esquema-procedimiento de las categorías de la cantidad es lo que Kant en el capítulo del esquematismo denomina «número» (Zahl). La síntesis del número consiste en una adición sucesiva de unidades homogéneas. El esquema-producto, en cambio, es la magnitud extensiva. La magnitud extensiva es una representación en la cual el todo es hecho posible por la agregación de partes homogéneas previamente dadas. La sección tercera de nuestro artículo se ocupa de establecer si el esquema de las categorías de la cantidad es una determinación del tiempo puro o una determinación temporal de lo empírico. Sostendremos que, pese a las apariencias en contra, el esquema de las categorías de la cantidad es una determinación temporal de la multiplicidad empírica.

\section{II}

Las categorías de la cantidad son la unidad, la pluralidad y la totalidad. Según Kant, es la misma función del entendimiento la que, por un lado, establece relaciones lógicas cuantitativas entre conceptos en juicios y, por el otro, sintetiza la multiplicidad de una intuición en conformidad con las mencionadas categorías 5 . En el primer caso, el entendimiento confiere a un juicio una forma lógica cuantitativa que determina que este juicio sea singular, particular o universal. Hasta aquí no nos encontramos con característica sensible alguna. Hay únicamente la indicación de que bajo el concepto sujeto caen uno, algunos o todos los elementos de una clase, y de que esos elementos están contenidos asimismo en la esfera del concepto predicado ${ }^{6}$. Aún no está establecido cómo estas determinaciones deben ser expresadas espacio-temporalmente ${ }^{7}$.

${ }^{5}$ Cf. A 79/B 104-105. Para una útil explicación del concepto kantiano de función, cf. Wolff (1995: 19 ss.). Cf. también Caimi (2000).

${ }^{6}$ Cf. Caimi (2009: 78). Hemos tenido en cuenta únicamente, a fin de simplificar la exposición, los juicios afirmativos.

${ }^{7}$ Como veremos más adelante, el esquema trascendental de la cantidad expresa intuitivamente el contenido de las categorías de la cantidad y de esa manera hace posible que éstas se vinculen con la multiplicidad empírica. Cf. infra, n. 32. 
En el segundo caso, la multiplicidad sintetizada por las categorías puede estar contenida en una intuición en general o en una intuición espacio-temporal de nuestra sensibilidad. Si la multiplicidad es espacio-temporal, la espontaneidad encargada de sintetizarla es la de la de imaginación. Esta síntesis de la multiplicidad espacio-temporal no puede tener lugar inmediatamente, sino que presupone la producción de un procedimiento universal de síntesis o esquema-procedimiento. Sin el correspondiente esquema-procedimiento, una categoría no es más que un concepto vacío incapaz de proporcionar conocimiento.

Por tanto, la multiplicidad empírica dada a nuestra sensibilidad no puede ser subsumida inmediatamente bajo las categorías de la cantidad. Esta subsunción requiere la mediación de un esquema trascendental. En el capítulo del esquematismo, Kant afirma que el esquema trascendental de las categorías de la cantidad es el número, y que el número «(...) es una representación que abarca la adición sucesiva de lo uno a lo uno (homogéneos) $»^{8}$. El número no es sino «(...) la unidad de la síntesis de lo múltiple de una intuición homogénea en general $(\ldots) »^{9}$.

Podemos extraer dos conclusiones de las líneas citadas. En primer lugar, hay un solo esquema para las tres categorías de la cantidad. No se trata de que sólo una de las categorías de la cantidad sea esquematizada, sino de que la imaginación produce un único esquema para las tres categorías en conjunto ${ }^{10}$. En el texto citado se afirma que el esquema de la cantidad viene dado por una síntesis o adición sucesiva de unidades homogéneas. Ahora bien, una adición sucesiva de unidades presupone la intervención de las tres categorías de la cantidad. Tal adición, en efecto, consiste en la síntesis sucesiva de una pluralidad de unidades, de modo tal que ellas conformen una totalidad. El número es el esquema de la cantidad en la medida en que en su producción entran en juego las tres categorías de la cantidad (unidad, pluralidad y totalidad). Por este motivo, el número hace posible la aplicación de las tres categorías mencionadas ${ }^{11}$.

${ }^{8}$ A $142 / B 182$.

${ }^{9}$ A $142-143 /$ B 182.

${ }^{10}$ Con respecto al problema de que los esquemas trascendentales no sean doce como las categorías, sino ocho, cf. Paton (1970: II, 63-65).

${ }^{11} \mathrm{El}$ hecho de que Kant llame "número" $(Z a h l)$ al esquema trascendental de las categorías de la cantidad no implica que ese esquema sea un número. La circunstancia de que el esquema que nos ocupa haya sido denominado de esa manera se debe, posiblemente, a que los esquemas de los 
La segunda conclusión que puede extraerse de la definición del esquema de la cantidad es que éste es presentado como un procedimiento. El esquema de la cantidad es el número, y éste consiste en la adición o síntesis sucesiva de unidades homogéneas. Es claro que estamos ante un procedimiento de síntesis o esquemaprocedimiento $^{12}$. A nuestro entender, los esquemas-procedimientos generan en la multiplicidad empírica propiedades temporales fundamentales (esquemas-productos). Un análisis de la síntesis llevada a cabo por el esquema-procedimiento de la cantidad nos permitirá arribar a una comprensión del correspondiente esquema-producto.

La definición ofrecida por Kant del número como esquema de la cantidad puede esclarecerse en función de lo expuesto en los pasajes dedicados al correspondiente principio del entendimiento. Por tanto, presentaremos sucintamente la prueba incluida en los "Axiomas de la intuición". A fin de ponernos en condiciones de comprender la prueba mencionada, brindaremos previamente un análisis sucinto de las intuiciones puras del espacio y el tiempo.

En la «Estética trascendental» se afirma que los distintos tiempos y espacios sólo pueden representarse, respectivamente, como partes del mismo tiempo ${ }^{13} \mathrm{y}$ del espacio único ${ }^{14}$. Por ejemplo, representamos necesariamente un metro cúbico de espacio dentro de un espacio ilimitado que lo rodea. El espacio y el tiempo son intuiciones porque, a diferencia de los conceptos, no contienen una multiplicidad bajo ellos mismos, sino en ellos mismos. Dicho en otras palabras, los tiempos y los espacios particulares son limitaciones del tiempo y el espacio únicos ${ }^{15}$.

En una nota a pie de página del $\$ 26^{16}$ se introduce el concepto de intuición formal. Kant afirma que el espacio y el tiempo como formas de la intuición sumi-

conceptos aritméticos, o conceptos de números, pueden entenderse como particularizaciones del esquema trascendental de las categorías de la cantidad. Cf. infra, n. 24.

${ }^{12}$ Cf. infra, n. 21.

${ }^{13}$ Cf. A 32/B 47.

${ }^{14}$ Cf. A 25/B 39.

${ }^{15}$ Que el tiempo sea infinito significa precisamente que cualquier magnitud determinada de tiempo sólo es posible como limitación del tiempo único. El tiempo único ilimitado sirve de fundamento para la representación de cualquiera de sus partes. Cf. A 32/B 47-48. Lo mismo puede afirmarse del espacio. Para una discusión del tema, cf. Chenet (1994: 82-87).

${ }^{16}$ Cf. B $160-161 \mathrm{n}$. 
nistran una multiplicidad. Tal multiplicidad no puede consistir sino en los espacios y tiempos particulares mencionados más arriba. El espacio y el tiempo como intuiciones formales, en cambio, presuponen una síntesis de esa multiplicidad. Esta síntesis es regida por un cierto concepto y ejecutada por la imaginación. De este modo es posible, por ejemplo, la representación de un espacio como objeto, es decir, un espacio determinado. Un espacio determinado es un objeto de intuición que presupone la unificación de partes del espacio único en conformidad con un concepto. Un ejemplo de espacio determinado es la representación de una figura geométrica tal como una línea recta ${ }^{17}$.

En los «Axiomas de la intuición» Kant comienza su prueba con la afirmación de que todos los fenómenos nos son dados en el espacio y en el tiempo. Por este motivo los fenómenos contienen una multiplicidad de partes. Posteriormente Kant nos recuerda que la representación de un tiempo o un espacio determinados presupone una síntesis de la multiplicidad de partes homogéneas del tiempo o el espacio. Kant concluye que los fenómenos, en tanto intuiciones en el espacio y el tiempo, no pueden ser aprehendidos sino mediante la misma síntesis sucesiva que determina el espacio y el tiempo ${ }^{18}$.

Por tanto, la intuición de un fenómeno presupone una síntesis de sus partes extendidas en el espacio y sucesivas en el tiempo. Los fenómenos son intuidos como agregados de partes previamente dadas. En otras palabras, los fenómenos son magnitudes extensivas. La magnitud extensiva es definida por Kant de la siguiente manera: «llamo magnitud extensiva a aquélla en la que la representación de las partes hace posible la representación del todo (y por consiguiente, precede necesariamente a ésta)» ${ }^{19}$.

Puede concluirse, aunque Kant no lo afirme, que la magnitud extensiva es el esquema-producto de las categorías de la cantidad ${ }^{20}$. El esquema-procedimien-

${ }^{17}$ Coincidimos en la interpretación de la intuición formal con Henry E. Allison. Cf. Allison (2004: 115-116).

${ }^{18}$ Cf. B 202-203.

${ }^{19}$ A 162/B 203.

${ }^{20}$ Podría objetarse que Kant no califica la magnitud extensiva como un esquema. No obstante, la concepción de los esquemas como propiedades o productos tiene sustento textual. Por ejemplo, los esquemas de las categorías dinámicas (relación y modalidad) son presentados no tanto como procedimientos, sino más bien como propiedades (cf. A 144-145/B 183-184). Los esquemas de las 
to de estas categorías es el número, «(...) una representación que abarca la adición sucesiva de lo uno a lo uno (homogéneos) ${ }^{21}$. Y una magnitud extensiva no es sino el resultado de una composición sucesiva de partes homogéneas ${ }^{22}$. Es claro que la síntesis del número (esquema-procedimiento) genera la magnitud extensiva (esquema-producto).

Las unidades homogéneas agregadas por la síntesis del esquema-procedimiento no son sino las partes espacio-temporales de los fenómenos. Tales partes son homogéneas en la medida en que se hace abstracción de sus determinaciones cualitativas. La síntesis en cuestión consiste en la adición sucesiva de una pluralidad de partes tomadas como unidades, y la posterior consideración de esta pluralidad como una unidad, de modo tal que se produzca una totalidad. La tota-

categorías matemáticas (cantidad y cualidad), en cambio, son expuestos en el capítulo del esquematismo como procedimientos. Las propiedades producidas por esos procedimientos se estudian en las secciones dedicadas a los principios del entendimiento correspondientes a esas categorías («Axiomas de la intuición»y «Anticipaciones de la percepción»). Por otro lado, la concepción del esquema como producto se pone en evidencia cuando Kant trata el esquema trascendental como «(...) un tercero (...)», «(...) representación mediadora (...)» (A 138/B 177) y «(..) un producto trascendental de la imaginación (...)» (A 142/B 181). Hasta donde sabemos, el primer comentarista en sostener que los esquemas trascendentales son características o propiedades de los objetos empíricos fue Paton. Cf. Paton (1970: II, 28-30, 43). Paton fue seguido por Wolfgang Detel y Klaus Düsing (Detel, 1978: 42-43; Düsing, 1995: 69). También Henry E. Allison, con algunas modificaciones, sigue esta interpretación (Allison, 1981: 70-71; 1983: 183; 2004: 217).

${ }^{21}$ En el capítulo del esquematismo de la Crítica de la razón pura Kant caracteriza el esquema como «(...) representación de un procedimiento universal de la imaginación para suministrar su imagen a un concepto (...)» (A 140/B 180-181). Ahora bien, esta caracterización es válida únicamente para los esquemas de los conceptos empíricos y matemáticos. El esquema trascendental o esquema de una categoría «(...) es (...) algo que no puede ser llevado a imagen alguna (...)» (A 142/B 181). Sin embargo, en Crítica de la razón práctica Kant se refiere al esquema trascendental como a un "procedimiento universal de la imaginación» (AA, V, 69). Hemos intentado mostrar que los esquemas son procedimientos de la imaginación regidos por reglas conceptuales categoriales en Arias Albisu (2010). Cf. también La Rocca (1989: 134-135, 152).

${ }^{22}$ Cf. A 162/B 202-203. En B 201-202 n. Kant afirma: «todo enlace (conjunctio) es, ya composición (compositio), ya conexión (nexus). La primera es la síntesis de lo múltiple [cuyos elementos] no se pertenece[n] necesariamente unos a otros, como p. ej. los dos triángulos en los que se divide un cuadrado mediante la diagonal, tomados por sí mismos, no se pertenecen necesariamente uno al otro; y tal es la síntesis de lo homogéneo en todo lo que puede ser considerado matemáticamente (síntesis que a su vez puede dividirse en la de la agregación y la de la coalición, de las cuales la primera se dirige a magnitudes extensivas, la otra a magnitudes intensivas).» La síntesis del esquema-procedimiento de las categorías de la cantidad es una síntesis de la agregación. Tal síntesis se dirige a elementos homogéneos que no se pertenecen necesariamente unos a otros. 
lidad en cuestión no es sino una magnitud extensiva. Puede apreciarse, en primer lugar, que las tres categorías de la cantidad rigen conjuntamente la síntesis del esquema-procedimiento que da lugar al esquema-producto. En segundo lugar, parece claro que las categorías de la cantidad, mediante su esquema-procedimiento, hacen que los fenómenos, espacialmente, posean ciertas anchura, largura y profundidad y, temporalmente, cierta duración ${ }^{23}$.

Hemos adelantado que los esquemas-productos son propiedades temporales fundamentales producidas en la multiplicidad empírica. Y, ciertamente, la magnitud extensiva es una propiedad temporal. El hecho de que los fenómenos sean intuidos como agregados de partes previamente dadas presupone que nuestra aprehensión de ellos es sucesiva. Si nuestra aprehensión de los fenómenos no fuese sucesiva, no podría haber una síntesis que componga una a una sus partes ${ }^{24}$.

Además, la magnitud extensiva es una propiedad fundamental, dado que define qué significa ser un objeto empírico en lo que a la dimensión cuantitativa de éste se refiere. Cuando la multiplicidad empírica presenta esa propiedad, es constituida como objeto empírico en lo que a su dimensión cuantitativa respecta. La formulación kantiana del principio del entendimiento correspondiente a las categorías de la cantidad muestra claramente que la magnitud extensiva es una propiedad fundamental de los objetos empíricos. Efectivamente, en ese principio la magnitud extensiva es atribuida a un aspecto del ámbito objetivo.

\footnotetext{
${ }^{23}$ Acerca de la conformación de la duración, cf. infra, n. 35.

${ }^{24} \mathrm{Si}$ bien no es nuestra intención tratar el complejo problema de la relación entre los conceptos matemáticos y las categorías y el esquema de la cantidad, nos permitimos presentar una hipótesis. Según se expresa Kant en $K r V$, los conceptos geométricos rigen una síntesis que consiste en un trazar o dibujar (ziehen, zeichnen, verzeichnen) parte a parte una figura (cf. A 162-163/B 203). Los conceptos aritméticos, en cambio, pueden regir dos diferentes acciones de síntesis. En primer lugar, tal acción puede ser un enumerar (zählen) una colección de intuiciones formales (como puntos y rayas) u objetos (como los dedos de la mano) tomados como unidades. En segundo lugar, la acción mencionada puede consistir en un medir (messen) un objeto, lo cual presupone la adopción de una unidad de medida y la constatación de cuántas veces está ella incluida en el objeto. (En la Reflexion No 5727 -AA, XVIII, 338- se da a entender que la determinación de magnitudes tiene lugar mediante «Messen und zählen»-medición y enumerar-). Parecería claro que los esquemas-procedimientos de síntesis correspondientes a los conceptos geométricos y aritméticos pueden entenderse como particularizaciones de la síntesis del esquema-procedimiento de las categorías de la cantidad. En todos esos casos, la síntesis consiste en un combinar sucesivamente unidades homogéneas.
} 
Según la edición de 1781 de $K r V$, el principio establece: «todos los fenómenos son, según su intuición, magnitudes extensivas» ${ }^{25}$.

\section{III}

La definición kantiana del esquema trascendental como «transscendentale Zeitbestimmung ${ }^{26}$ puede comprenderse, ante todo, de dos maneras diferentes. ${ }^{27}$ En primer lugar, «Zeitbestimmung» puede traducirse por «determinación del tiempo». En este caso, el esquema trascendental consiste en una determinación de la multiplicidad pura de la intuición del tiempo en conformidad con las categorías. En segundo lugar, «Zeitbestimmung» puede entenderse como «determinación temporal». En este caso, el esquema trascendental es una determinación temporal de la multiplicidad empírica espacio-temporal de acuerdo con las categorías $^{28}$. La tercera sección de este artículo está dedicada a mostrar que el esquema trascendental de las categorías de la cantidad no es una determinación de la intuición pura del tiempo, sino una determinación temporal de lo empírico ${ }^{29}$.

${ }^{25}$ A 162.

${ }^{26}$ A $138 /$ B 177.

${ }^{27}$ Cf. p. 244, n. 525 de la traducción de Mario Caimi de la Crítica de la razón pura. Este punto fue tratado con cierta amplitud en Caimi (2004: 7 ss.)

${ }^{28}$ Herbert J. Paton, Henry E. Allison, Klaus Düsing y Markku Leppäkoski han afirmado de manera general que el esquema trascendental no consiste en una determinación del tiempo puro, sino en una determinación temporal de lo empírico espacio-temporal. (Cf. Paton, 1970: II, 2830; Allison, 1981: 70-71; 1983: 183; 2004: 217; Düsing, 1995: 69 y Leppäkoski, 1995: 17 ss.). Ninguno de estos comentaristas, salvo tal vez parcialmente Paton, se ha ocupado de mostrar la validez de esta tesis para el caso del esquema de las categorías de la cantidad. De hecho, Düsing sostiene que la tesis en cuestión es válida para todos los esquemas, excepto el de la cantidad, dado que éste posibilita ciertamente la matemática, pero esta última no constituye todavía conocimiento de la naturaleza empírica.

${ }^{29}$ El hecho de que los esquemas sean determinaciones temporales de lo empírico no implica que ellos no sean a priori. Intentaremos demostrarlo a continuación. Un conocimiento «a priori» es aquel conocimiento independiente, en cuanto a la validez, de la experiencia en general. (Los conocimientos a priori son independientes en cuanto a su validez de toda experiencia. En otras palabras, preceden lógicamente a la experiencia. Ahora bien, todo conocimiento comienza temporalmente con la experiencia. No habría ningún tipo de conocimiento si nuestras facultades cognoscitivas no tuvieran a su disposición intuiciones empíricas. Cf. B1-B2. Cf. la discusión del tema en Paton, 1970: I, 76-80). Además, Kant señala en más de una ocasión que los caracteres distintivos y suficientes del conocimiento a priori son la universalidad y la necesidad (cf. B 3-4, A 47/B 
A primera vista, nuestro examen del esquema trascendental de la cantidad, expuesto en la sección anterior, parece estar de acuerdo con la interpretación de «Zeitbestimmung» como «determinación del tiempo». Hemos visto que los fenómenos constan de una multiplicidad de partes en virtud de estar dados en el espacio y en el tiempo. Por este motivo, afirma Kant, deben ser representados por la misma síntesis que determina el espacio y el tiempo en general ${ }^{30}$. Parecería entonces que la síntesis del esquema-procedimiento de la cantidad toma por objeto la multiplicidad pura del espacio y el tiempo en general y permite representarla como magnitud extensiva ${ }^{31}$. Los fenómenos serían deter-

64, A 823/B 851). Consiguientemente, una determinación a priori debe consistir en una determinación universal y necesaria. Los esquemas trascendentales parecen cumplir estos requisitos. Son determinaciones universales, dado que tienen lugar en conformidad con las categorías y se dirigen a la multiplicidad empírica en general. Son también determinaciones necesarias, pues constituyen condiciones de posibilidad de nuestra experiencia de objetos empíricos constituidos como tales. No hay contradicción, entonces, entre nuestra interpretación de los esquemas trascendentales y la atribución a éstos de un carácter a priori. Cabe destacar que Kant también caracteriza el esquema como "puro» (rein, A 138/B 177). Sin embargo, como señala Paton, Kant a menudo utiliza "puro» como sinónimo de «a priori» (Paton, 1970: I, 76-77). Por último, Kant también califica al esquema como «trascendental» (cf. A 138/B 177). Trascendental es el conocimiento a prio$r i$ mediante el cual conocemos que ciertas representaciones son a priori y pueden referirse a priori a objetos de la experiencia. El conocimiento trascendental concierne por tanto a la posibilidad del conocimiento a priori y a la explicación y fundamentación de su validez objetiva (cf. A 56/B 8081). Esta aproximación al concepto «trascendental» no es enteramente aplicable a nuestro caso, pues los esquemas trascendentales no son conocimientos. Sólo podemos hablar de conocimiento en el nivel del juicio, y los esquemas no son más que condiciones para el juicio trascendental o principio del entendimiento puro (tales juicios expresan la subsunción de la multiplicidad empírica bajo las categorías posibilitada por los esquemas trascendentales. Cf A 136/B 175). Sin embargo, teniendo en cuenta la explicación precedente, podríamos afirmar que los esquemas son trascendentales en la medida en que constituyen una condición a priori del conocimiento a priori. Pues los esquemas trascendentales son independientes de la experiencia y posibilitan un conocimiento de hecho a priori, a saber, los principios del entendimiento puro (cf. A 148/B 187 ss.). Esta interpretación es confirmada por un pasaje del $\$ 24$ de $K r V$, en el que se afirma que la síntesis intelectual y la síntesis figurativa, que ciertamente no son todavía conocimientos, son llamadas trascendentales «(...) no solamente porque ellas mismas proceden a priori, sino también porque fundamentan la posibilidad de otro conocimiento a priori.» B 151.

30 "(...) es decir, todos los fenómenos son magnitudes, y magnitudes extensivas, porque, como intuiciones en el espacio o en el tiempo, deben ser representados por medio de la misma síntesis por la cual son determinados el espacio y el tiempo en general.» B 203.

${ }^{31}$ El principio de los «Axiomas de la intuición» de 1781 es «todos los fenómenos son, según su intuición, magnitudes extensivas». Esta formulación fue modificada en 1787. Según la segunda edición de $K r V$, el principio en cuestión es "todas las intuiciones son magnitudes extensivas». Heiner $\mathrm{F}$. Klemme señala con acierto que la edición de 1781 limita erróneamente el principio al ámbito de 
minados mediatamente como magnitudes extensivas en la medida en que son acogidos en las formas de la intuición sintetizadas por las categorías. La síntesis del esquema-procedimiento no se dirigiría a la multiplicidad empírica, sino que enlazaría inmediatamente la multiplicidad pura y sólo por intermedio de esta composición determinaría la multiplicidad empírica. El esquema trascendental de la cantidad no sería entonces una determinación temporal de la multiplicidad empírica. Parecería más apropiado comprenderlo como una determinación de la intuición pura del tiempo.

Notemos que la distinción efectuada por Kant entre categorías matemáticas y dinámicas parece aportar evidencia textual a favor de la interpretación del esquema de la cantidad como determinación del tiempo. Kant considera que las categorías de la cantidad y la cualidad son matemáticas, mientras que las de la relación y la modalidad son dinámicas. Nos interesa señalar que en B 110 Kant afirma que las categorías matemáticas se dirigen «(...) a objetos de la intuición (tanto de la pura, como de la empírica) (...)». Las categorías de la cantidad pertenecen al grupo de las categorías matemáticas. Por tanto, Kant ha afirmado con toda claridad que las categorías de la cantidad también se aplican a la intuición pura. El pasaje citado podría interpretarse en el sentido de que las categorías de la cantidad se dirigen inmediatamente a la intuición pura y sólo por medio de ésta a la intuición empírica.

De acuerdo con los textos citados anteriormente, parecería que la interpretación de "Zeitbestimmung» como «determinación temporal» no es válida con respecto a las categorías de la cantidad. Sin embargo, esta tesis no es correcta. A continuación procuraremos demostrar que, pese a las apariencias en contra, el esquema de las categorías de la cantidad debe ser comprendido como una determinación temporal de la multiplicidad empírica.

En primer lugar, sería arbitrario denominar «determinación del tiempo»al esquema de la cantidad en virtud de que se dirige a la intuición pura del tiempo. En efecto, hemos mostrado que las categorías de la cantidad también se aplican a la intuición pura del espacio. Por tanto, hubiera sido igual de legítimo definir este esquema como «determinación del espacio». En síntesis, si el esquema

los fenómenos, y que la modificación de la segunda edición apunta a establecer que no sólo las intuiciones empíricas, sino también las intuiciones puras son magnitudes extensivas. Cf. Klemme (1998: 257). 
de la cantidad fuese una determinación del tiempo, no se comprendería el privilegio concedido al tiempo en detrimento del espacio.

En segundo lugar, hay buenas razones para considerar el esquema de la cantidad como una determinación temporal. Este esquema es temporal por dos motivos. Por un lado, la síntesis del esquema-procedimiento de la cantidad consiste en una adición sucesiva de unidades homogéneas. Si la síntesis no tuviese lugar de forma sucesiva, y por tanto temporal, los fenómenos no serían magnitudes extensivas.

Por otro lado, el esquema-procedimiento de la cantidad hace posible la representación de los objetos empíricos como dotados de una determinación temporal, a saber, la duración. Así como la cantidad entendida espacialmente consiste en la anchura, la largura y la profundidad, la duración no es sino la cantidad interpretada temporalmente ${ }^{32}$. En su examen del principio del entendimiento correspondiente a la categoría de inherencia y subsistencia, Kant afirma: «Sólo gracias a lo permanente recibe la existencia en diversas partes sucesivas de la serie temporal una magnitud, que se llama duración. Pues en la mera sucesión solamente, la existencia está siempre desapareciendo y comenzando, y nunca tiene la más mínima magnitud ${ }^{33}{ }_{\text {». }}$

Ofreceremos únicamente una lectura preliminar del pasaje citado. El tiempo puro consiste en una sucesión de instantes efímeros. Las partes del tiempo son

${ }^{32}$ Los objetos poseen ciertas cantidades de anchura, largura y profundidad, así como cierta cantidad de duración, en tanto que son magnitudes extensivas (acerca de la conformación de la duración, cf. infra, n. 35). Por tanto, la magnitud extensiva, esquema-producto de las categorías de la cantidad, puede considerarse como la expresión intuitiva de esas categorías. Por este motivo la magnitud extensiva permite vincular las categorías de la cantidad con las intuiciones empíricas humanas y de esta manera posibilita la aplicación de las primeras a las segundas. Cabe destacar que el hecho de que la magnitud extensiva posea rasgos espacio-temporales no implica que el esquema trascendental de la cantidad sea esencialmente espacio-temporal y no simplemente temporal. Como hemos visto más arriba, el esquema trascendental de las categorías de la cantidad debe ser comprendido como una determinación temporal de la multiplicidad empírica espacio-temporal. El esquema-procedimiento de la cantidad consiste en una adición sucesiva de partes espacio-temporales de los fenómenos tomadas como unidades homogéneas. Tal adición o síntesis, en tanto sucesiva, es esencialmente temporal. Y el hecho de que la magnitud extensiva (esquema-producto de la cantidad) sea un agregado de partes previamente dadas se debe a ese carácter esencialmente temporal de la síntesis que la produce. Cf. infra, n. 40.

${ }^{33}$ A 183/B 226. 
sucesivas y no hay nada permanente en ellas. Lo permanente, según Kant, no puede ser sino algo efectivamente existente en el espacio ${ }^{34}$. Y sólo mediante lo permanente se hace posible la representación de una duración. Por tanto, consideramos que el texto establece que sólo en virtud de la existencia en el espacio de algo permanente a través del tiempo reciben los objetos empíricos una magnitud temporal, a saber, duración. La duración no es entonces una propiedad del tiempo puro que se derive de la determinación del mismo, sino una propiedad temporal de los objetos empíricos que presupone la existencia de algo permanente en el espacio. Ahora bien, la duración no es sino la magnitud extensiva temporal, y la magnitud extensiva es el esquema-producto de las categorías de la cantidad. En consecuencia, el esquema-producto de las categorías de la cantidad no es una propiedad del tiempo puro, sino una propiedad temporal de los objetos ${ }^{35}$.

Hemos mostrado que el esquema de las categorías de la cantidad no es una determinación de la intuición pura del tiempo, sino una determinación temporal de la multiplicidad empírica. Ahora bien, si éste fuera el caso, debemos explicar el significado de la afirmación kantiana de que las categorías de la cantidad se dirigen no sólo a la intuición empírica, sino también a la intuición pura ${ }^{36}$. Citamos más arriba el pasaje de B 203 donde se establece que los fenómenos deben ser representados «(...) por medio de la misma síntesis por la cual son determinados el espacio y el tiempo en general.» Hemos sostenido que esta tesis puede comprenderse en el sentido de que la síntesis en cuestión se dirige a la intuición pura y sólo por intermedio de ella a la intuición empírica. Ahora bien, la mencionada interpreta-

${ }^{34}$ Cf. B 275-276 y B 291.

${ }^{35}$ El esquema de la categoría de inherencia y subsistencia es la permanencia de lo real en el tiempo (cf. A 144/B 183). La duración, como hemos visto, presupone esta permanencia. Por tanto, podría objetársenos que la atribución de duración a los objetos empíricos no resulta de la aplicación de las categorías de la cantidad, sino de la aplicación de la categoría de inherencia y subsistencia. Consideramos empero que también las categorías de la cantidad intervienen en la producción de la magnitud temporal o duración. En efecto, el carácter mensurable de la duración se debe a que ella contiene una multiplicidad de partes. Las partes de una duración determinada pueden interpretarse como los momentos sucesivos durante los que existe un objeto permanente en el espacio. A fin de que un objeto posea una cierta duración, las partes en cuestión deben ser aprehendidas sucesivamente. Ahora bien, la aprehensión sucesiva de partes no es sino la síntesis del esquema-procedimiento de las categorías de la cantidad. Por consiguiente, la representación de objetos empíricos como dotados de una duración mensurable depende tanto del esquema de la substancia y el accidente como del esquema de las categorías de la cantidad.

${ }^{36}$ Cf. supra y B 110. 
ción no es la única posible. También puede afirmarse que la multiplicidad pura y la empírica son sintetizadas conjuntamente por el esquema-procedimiento de las categorías de la cantidad. Según esta interpretación, una única síntesis enlazaría la multiplicidad empírica en cuanto al espacio que ocupa y el tiempo en el que se presenta. Tal síntesis no reuniría el espacio y el tiempo en su totalidad ${ }^{37}$, sino que generaría espacios y tiempos determinados mediante la reunión de las multiplicidades empíricas dadas en tales espacios y tiempos ${ }^{38}$.

En síntesis, no es válida la tesis de que el esquema de las categorías de la cantidad consista meramente en una determinación del tiempo puro. No se trata de que la síntesis del esquema-procedimiento determine el tiempo puro en su conjunto y por intermedio de ello la multiplicidad empírica. Más bien, la síntesis en cuestión reúne la multiplicidad empírica en tanto ocupa espacio y se presenta en un tiempo ${ }^{39}$.

${ }^{37}$ Es evidente que no es posible representar ni el espacio ni el tiempo en su totalidad como magnitudes extensivas. En efecto, dado que tales intuiciones son ilimitadas, la síntesis de la agregación de sus partes requeriría un tiempo infinito para llevarse a cabo.

${ }^{38}$ Henry Allison señala acertadamente que los «Axiomas de la intuición» no se ocupan de la unificación del espacio único e ilimitado, sino de la «(...) representación de espacios determinados y de la conexión entre tal representación y la intuición de objetos en esos espacios». Allison considera además que la síntesis sucesiva que genera espacios determinados presupone «(...) que sean dadas las porciones homogéneas de espacio y, por tanto, que sea dado el espacio único omniabarcador del cual ellas son partes.» Allison, 1983: 95-96. Consideramos que la tesis de Allison también es aplicable al caso del tiempo.

${ }^{39}$ La síntesis en cuestión se dirige a la intuición empírica. Las formas de tal intuición son el espacio y el tiempo. Por otra parte, la materia de la intuición viene dada por la sensación. Kant ha afirmado explícitamente que una única síntesis se dirige al compuesto de materia y forma en que consiste la intuición empírica. «Por consiguiente, la intuición empírica no está compuesta de fenómenos y de espacio (de percepción y de intuición vacía). Lo uno no es correlato de lo otro en la síntesis, sino que [ambos] sólo están enlazados en una y la misma intuición empírica, como la materia y la forma de ella.» A 429/B 457 n. (Señalemos que el pasaje citado se encuentra en una nota a la observación a la antítesis de la primera antinomia. La circunstancia de que este pasaje se encuentre en la observación al argumento de la antítesis y no en el argumento mismo indica que aquél no expresa la concepción de la antítesis, sino que refleja el pensamiento de Kant mismo. Coincidimos con Lorne Falkenstein en que el pasaje citado es relevante para la comprensión del pensamiento de Kant. Cf. Falkenstein, 1995: 84-87). Sin embargo, recordemos que la síntesis del esquema trascendental de las categorías de la cantidad se dirige a partes homogéneas. Por tanto, la síntesis en cuestión presupone que se haga abstracción de las diferencias materiales o cualitativas de los elementos de la multiplicidad empírica. Tal síntesis reúne la multiplicidad empírica espacio-temporal, pero hace abstracción de las diferencias cualitativas entre los elementos de tal multiplicidad. En conclusión, la síntesis del esquema trascendental de las categorías de la cantidad no se dirige meramente a una forma pura, sino a un compuesto de materia y forma en su dimensión formal. 
Por tanto, el esquema trascendental de las categorías de la cantidad bien puede ser comprendido como una determinación temporal de la multiplicidad empírica $^{40}$.

A fin de concluir el presente artículo, procuraremos explicar una críptica declaración de Kant en función de la discusión precedente. Tal declaración fue incluida por Kant en la descripción del esquema de las categorías de la cantidad. Kant afirma lo siguiente: «(...) el número no es otra cosa que la unidad de la síntesis de lo múltiple de una intuición homogénea en general, de modo tal que produzco el tiempo mismo en la aprehensión de la intuición ${ }^{41}$. Nos interesa destacar la indicación de que mediante la síntesis del esquema-procedimiento de la cantidad (número) producimos el tiempo mismo en la aprehensión de la intuición. Según lo mostrado más arriba, la tesis de que el tiempo es producido no puede significar sino que en la aprehensión de la multiplicidad empírica se generan tiempos determinados. El esquematismo de las categorías de la cantidad posibilita de esta manera la representación de una serie de objetos dotados de una cierta duración o, en otras palabras, una serie temporal. ${ }^{42}$

${ }^{40} \mathrm{El}$ hecho de que los esquemas trascendentales sean determinaciones de lo empírico espacio-temporal no implica que ellos mismos sean esencialmente espacio-temporales. Nos oponemos así a Gregg E. Franzwa. Según Franzwa, diversos pasajes de la segunda edición de $\mathrm{KrV}$ demuestran que Kant debería haber modificado su doctrina de los esquemas como «determinaciones trascendentales del tiempo" y habernos ofrecido una explicación espacio-temporal de los mismos (cf. B 291 y Franzwa, 1978). Ahora bien, las tesis de Franzwa presuponen una interpretación de "Zeitbestimmung» como determinación del tiempo puro. En efecto, si éste fuera el caso, no se entendería la presencia de elementos espaciales en las descripciones kantianas de los esquemas trascendentales. Nosotros, en cambio, entendemos "Zeitbestimmung» como determinación temporal. Por este motivo sostenemos que los esquemas trascendentales son determinaciones temporales de la multiplicidad empírica espacio-temporal. No hay contradicción, entonces, entre nuestra concepción de los esquemas y el hecho de que el esquema-procedimiento de las categorías de la cantidad sintetice en el tiempo la multiplicidad empírica en tanto ocupa espacio y se presenta en un tiempo.

${ }^{41}$ A $142-143 /$ B 182.

${ }^{42}$ En A 145/B 184 Kant establece que el esquema trascendental de las categorías de la cantidad se refiere a «(...) la serie del tiempo (...)». 


\section{Bibliografía}

\section{Fuentes y traducciones}

KANT, I. (1902 ss.). Kant's gesammelte Schriften, Berlin, hrsg. von der Königlich Preußischen Akademie der Wissenschaften (AA).

Kant, I. (2007). Crítica de la razón pura (trad. de M. Caimi), Buenos Aires, Colihue.

\section{Bibliografía secundaria citada}

Allison, H. E. (1981). "Transcendental Schematism and the Problem of the Synthetic A Priori», Dialectica, 35, 1-2, pp. 57-83.

- (1983). Kant's Transcendental Idealism. An Interpretation and Defense, New HavenLondon, Yale University Press.

- (2004). Kant's Transcendental Idealism. Revised and Enlarged Edition, New Haven/London, Yale University Press.

ARIAS ALBISU, M. (2009). «Una relación de homogeneidad entre términos heterogéneos. El concepto de homogeneidad en el capítulo del esquematismo de la Crítica de la razón pura», Diánoia, 54, 63, pp. 71-88.

- (2010). «Los esquemas trascendentales como procedimientos y productos», Revista de filosofia (Madrid), 35, 2, pp. 27-42.

CAIMI, M. (2000). «Einige Bemerkungen über die Metaphysische Deduktion in der Kritik der reinen Vernunft", Kant Studien, 91, 3, pp. 257-292.

- (2004). Seminario sobre esquematismo (grabaciones inéditas del seminario de grado titulado «Kant. El esquematismo» y dictado en la Facultad de Filosofía y Letras de la Universidad de Buenos Aires durante el primer cuatrimestre de 2004; manuscrito cedido por el autor el 20/08/2006).

- (2009). "The Schema of Quantity», en A. Leirfall y T. Sandmel (eds.), Enhet i mangfold. Festskrift til Johan Arnt Myrstad $i$ anledning 60-årsdagen, Oslo, Unipub, pp. 7795.

CHENET, F. X. (1994) L'assise de l'ontologie critique: l'Esthétique transcendantale, Lille, Presses Universitaires de Lille.

DETEL, W. (1978) «Zur Funktion des Schematismuskapitels in Kants Kritik der reinen Vernunft», Kant Studien, 69, 1, pp. 17-45. 
DÜSING, K. (1995). «Schema und Einbildungskraft in Kants Kritik der reinen Vernunft», en L. Kreimendahl (ed.), Aufklärung und Skepsis. Studien zur Philosophie und Geistesgeschichte des 17. und 18. Jahrhunderts. Günter Gawlick zum 65. Geburtstag, Stuttgart-Bad Cannstatt, Frommann-Holzboog, pp. 47-71.

FALKENSTEIN, L. (1995). Kant's Intuitionism. A Commentary on the Transcendental Aesthetic, Toronto, University of Toronto Press Incorporated.

FRANZWA, G. E. (1978). «Space and the Schematism», Kant Studien, 69, 2, pp. 149159.

KLEMME, H. F. (1998). «Die Axiome der Anschauung und die Antizipationen der Wahrnehmung», en G. Mohr y M. Willaschek (eds.), Immanuel Kant: Kritik der reinen Vernunft, Berlin, Akademie, pp. 247-266.

LA ROCCA, C. (1989). «Schematismus und Anwendung», Kant Studien, 80, 2, pp. 129-154.

LEPPÄKOSKI, M. (1995). «The Transcendental Schemata», en H. Robinson (ed.), Proceedings of the Eight International Kant Congress, II, 1, Milwaukee, Marquette University Press, pp. 13-20.

LONGUENESSE, B. (2000). Kant and the Capacity to Judge (trad. de C. T. Wolfe), Princeton, Princeton University Press.

PATON, H. J. (1970). Kant's Metaphysic of Experience. A Commentary on the First Half of the Kritik der reinen Vernunft, London, George Allen \& Unwin Ltd.

WOLFF, M. (1995). Die Vollständigkeit der kantischen Urteilstafel. Mit einem Essay über Freges Begriffsschrift, Frankfurt am Main, Vittorio Klostermann.

Recibido: 15/03/2011

Aceptado: 19/04/2011 